\begin{tabular}{|c|c|}
\hline $\begin{array}{l}\text { MINING AND METALLURGY INSTITUTE BOR } \\
\text { UDK: } 622\end{array}$ & $\begin{array}{l}\text { ISSN: 2334-8836 (Štampano izdanje) } \\
\text { ISSN: 2406-1395 (Online) }\end{array}$ \\
\hline UDK: $681.51^{\prime} 71^{\prime}(045)=111$ & doi: $10.5937 / \mathrm{mmeb} 2002057 \mathrm{~T}$ \\
\hline
\end{tabular}

\title{
DEVELOPMENTAL TENDENCIES FOR THE DIGITAL PERSPECTIVE
}

\begin{abstract}
Development processes are ongoing. Jumping growth and development in the social systems is not possible. It is possible to accelerate the technological and technological progress in accordance with the evolutionary laws of gradualism. The existence of created values throughout the history of creation must correspond in a harmonized form with nature. More knowledge and less distrust is the formula for the survival of human civilization. Rational intentions of survival and duration are the results, not profits. Is the ecological movement a revolt against a conspiracy to destroy natural resources and its natural laws based on the evolutionary functioning? For the purpose of active response, it appears that the material aspect of development will increasingly be replaced by the non-material and innovative achievements. What is in sight: intellectual capitalism or the destructive power of mind and capital? Due to the fragmentation of knowledge, we have to rediscover the theory of systems and systems approach, as well as cybernetics. A comprehensive, interdisciplinary approach to innovative issues emerges as the first scientific, professional and systemic prerequisite for the success of a business in which an organization is intent on innovating. Due to the fact that the evolutionary trends, including digitalization, have led to the significant revisions of many current understandings, research into these topics is linked to accelerating the materialization of the scientific and technical developments. Industry 4.0 happened to us. That is not a surprise. These are the evolutionary processes that are a function of the emergence and disappearance of certain technologies (mechanical, energy, informatics) on the principle of the dual effect of technical progress: affirmative and devaluing. Will digitization squeeze out a market like the market eliminated feudalism? The digital perspective comes to us at the speed of acceleration (the first derivation of the velocity vector). It is the result of innovative processes that take place under the evolutionary laws. It is a symbiosis of information, cyber and digitized structures as the paradigms of digital perspective. The new non-industrialized economies have emerged and will emerge that will characterize the coming time. Every economy will strive to create the elements of its protection through the power of appearances or the laws of defense.
\end{abstract}

Keywords: innovation; evolution; digitalism; the future; technology; technical progress.

\section{INTRODUCTION}

We start from the fact that the principles of operation of the process of evolution, although not yet thoroughly researched and clarified, and achievements in relation to the natural organ systems are almost universally accepted and socio-economically implemented.

Contrary to the organ systems, their emergence and evolutionary survival (continuous adaptation and active feedback), the

\footnotetext{
*University of Pristina, Faculty of Economics in Kosovska Mitrovica,

e-mail: snezana.todosijevic1@ @otmail.com

** Higher Medical School of Professional Studies "Medika", Belgrade,

e-mail: zorankatanic@yahoo.com

**** e-mail: milos.todosijevic@hotmail.com

**** Full professor, University “Union Nikola Tesla, Mladenovac,Belgrade,damnjar@mts.rs
} 
problems of socio-cultural evolution are more controversial, since it is shown that the existence of purposefully rational social organizations is attributed solely to the rational human action on the basis of planning.

The difference is that the natural systems are created by a spontaneous order, social organizations are regarded as made, constructed order. This observation is applied in particular to an enterprise that we regard as an efficient and meaningful creation, shaped by the organizational structure of its structure based on goal-directed action. Companies, in terms of their size and complexity in organizational terms, are the result of rational behavior of decision makers and therefore numerous and varied forms of production and business cooperation, depending on the degree of complexity of product development.

A holistic, interdisciplinary approach to the innovative issues emerges as the first scientific, professional, and systemic prerequisite for the success of a business in which an organization intends to innovate. The reservoir of knowledge is a consequence of the educational process. Assumptions are created from total human resources to create a critical - desirable level of user and knowledge creator. The task of higher education institutions is not to recruit, but to produce from that production, to make the positive scientific selection to increase the power of knowledge.

Trade competitiveness and the role of technological innovation and technological transfer are becoming a central issue of an enterprise growth and development today, as the core of modern economic development is precisely the phenomenon of innovation. Its formation requires a lot of prior knowledge, which means that the laws of evolution still work here. Knowledge grows much like a population. The population grows in proportion to the last generation. Knowledge grows in proportion to the mass of knowledge left by the last generation. And what is impossible for science and innovation!? Upgrades are continuous both in the form of achievements and in shortening the time of realization. Influenced by the Industry 4.0; We have defined a slogan that is generally accepted: "who adjusts will last, who does not adjust will disappear." The acceleration is contributed by the information technology and digitalization-based processes. When to react? Immediately! Because if not us - who? If not now - When? A slogan we have taken from a decision theory.

It is only under the assumption of their own innovative capacity that it is possible to ensure satisfactory development performance and a competitive position in the world market. In this context, the essence of innovation management, understood, points to the conclusion that its basic task is not to develop the science and technology "per se", but to put the scientific and technological solutions into the function of economic and even wider social development [11].

The onset of technology and innovation-driven change necessitates the need for the new technology for managing the complex dynamic systems. Management technology is the oldest (created long before mechanical, energetic and informatics) and it emerges as the key to everything, even business success.

We recognize two major types of technological change: quantum and developmental (incremental). Quantitative (quantum) technological change is a fundamental shift in technology that results in innovation the new types of goods and services. Two examples of such technological change are the development of the Internet, which has revolutionized the computer industry, and the development of genetic engineering (biotechnology), which promises a revolution in the treatment and treatment of diseases of medicines so made that have the characteristic of smart drugs, because they focus their activity only on the diseased place. McDonald's development of the fast food supply principle is also an example of a quantitative technological shift. In a world 
of growing insecurity, change is a direction with no clear purpose [2]. As the nomadic tribes, contemporary companies, within their capabilities, define their development for a competitive level, while in the external environment they move from market to market, seeking a position to maintain their capabilities. Winners are those who have the knowledge and energy to develop the exceptional abilities and values for particular parts of the market. Measures of business success are shown at the right time by the finances.

We are on the threshold of a fundamental achievement. It was recently reported that Google had indeed achieved quantum supremacy, which would mean that it had the "unlocked" the ability of quantum computers to solve problems that the classic computers either failed or needed much more time.

The anticipated success in this area would lead to a turning point and an incentive in the field of quantum machines, as they would achieve the set goals much faster. Their tremendous processing power could help find new smart drugs, medical solutions, new materials, and create much more efficient supply chains and lead to a highly advanced artificial intelligence. ${ }^{\dagger}$

\footnotetext{
* In 2018, Google agreed with NASA to use their supercomputers as a benchmark for their quantum power experiments. According to the Financial Times, the report said that Google's quantum processor was able to complete in three minutes and 20 seconds the calculations that take about 10,000 years in today's most advanced supercomputer, the Summit. In a withdrawn report, experts said that "the trial represents the first computation that can only be performed by a quantum processor."

Quantum computers are so powerful because they use "quantum bits", so-called qubits. Unlike the usual bits, which are either 1 or 0 , cubic, in simplistic terms, can be both at the same time. Thanks to the quantum phenomenon, quantum computers can simultaneously process huge amounts of information, while traditional computers have to process them sequentially or gradually. Such success would lead to much needed incentives in the field of quantum machinery.
}

The evolutionary trends that include digitalization have led to significant revisions of many current understandings. Radical change is happening from an evolutionary perspective. This perspective reminds me of modesty, restraint and thinking about the limits of the possible. Cyber systems always inaugurate a control. This is also true of leadership as the most important social function of shaping.

The digitalization of the economic and social sectors, with the use of modern technologies in business processes, raises the issue of information and cyber security of the digitalized structure and achieves a significant development in this direction.

"Cognitive cybernetics is a new scientific discipline that, on the basis of cognitive sciences and cognition as a mental human charge, connects cybernetic understanding of everything as a system (technical, social, natural,) with the new intelligent technologies to point to the knowledge of interaction on the captological anthropomorphization of today's man"[1]. From the point of view of the operation of general laws, economics of social work, the fields of science and education are the levers of accelerated development of productive forces, and through the culture of work and production relations in the processes and processes of expanding the material basis of labor.

\section{INNOVATION CHANGES AND PROCESSES}

The world economy is undergoing the "tectonic shifts" and "floods and transformations". This is due to the fact that there is no universal model for change, and that there is no recipe [17].

The general methodology of innovation technology consists of three parts: system identification; system analysis; and research analysis [5]. 
The methodology of innovation technology is derived through a system of projects that are interdisciplinary linked to the specific scientific fields and technologies.

Education is an intermediary in understanding and applying the scientific out puts and "strengths" of personnel in resource management. In fact, the science and education are "the germs of growth that must be fermented in every human activity today [6]."

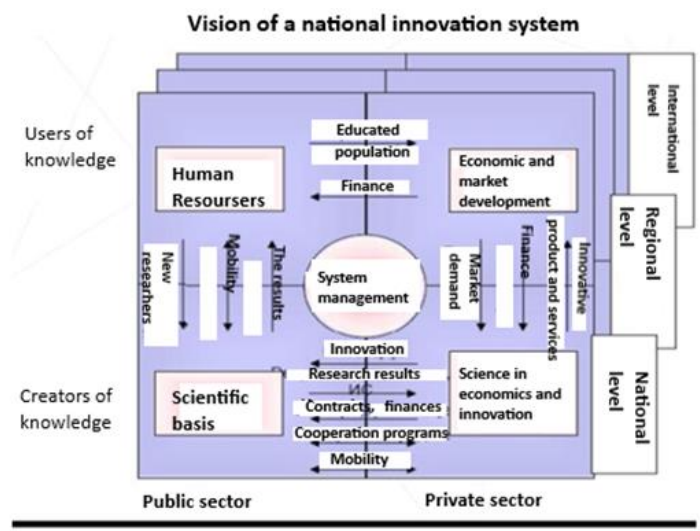

Figure 1 A general model of innovative activity (UNESCO's General Model for Innovative Activity)

There is no absolute progression without relative recourse. With the new knowledge, obsolescence comes into being and also the second-class position of the existing knowledge is impaired. This implies the need for so-called life-long education, continuing education and work-to-work education (return education). The authors of this paper have long asked the following question on this topic: "tell us how you spend your free time and let us tell you who you are"? The answer is, "Learning is eternal." On the other hand, by their economic functions, the education and science are characterized by the work of reproduction. Investments in science and education are proving to be the most profitable investment both in terms of return period and quality of output [15].

The technology advancement strategy, and the overall economic environment, is basically an absorbing milieu for implementing the results of research and development activities. It is about knowledge. In technological development there is a basic premise for more favorable international economic relations as well as for the policy of innova- tion and independence, which are always the requirements of a progressive society.

In all rich industrial nations, more and more people are worried about rising unemployment and stagnant wages. The alleged causes are globalization and amazingly rapid technological change. Computers and robots, along with the management techniques, are said to destroy jobs. At the same time, the free flows of trade and capital and huge advances in telecommunications have increased the international competition (competition) and made it easier for firms to transfer production to the developing countries with low wages. Differences between rich and poor are increasing, and drastic reduction of differences is impossible because the rich find ways to protect their own interests. Many feel that Adam Smith's invisible hand is trying to push them off a cliff. It is doubtful that today's government policies are no longer functioning as they are not suitable for the new global, digital economy [4]. The general question that may be asked is: Does the technological revolution also require an economic revolution? 
Permanent training of employees and expertly oriented teams are a function of precondition for the successful business. The goal is not profit but result. The level of modernity of business organizations and other economic entities presupposes the modernization of the business operations of enterprises, as well as of all other sectors of the large social and economic system, due to the causal connection. Such behaviors are merely a response to the need to survive and last, which is the primary condition of evolution.

Industry 4.0, dubbed the Fourth Industrial Revolution, although content-wise it is neither industry nor revolution, accelerated change but also responded as a challenge to those changes, which radically disrupted but also directed and mobilized organizational and technical-technological efforts to meet the challenges of this of the oncoming boom. It is an evolutionary process of developing science and technology, knowledge, as part of the totality of the cyber economic milieu [13].

Over the last twenty-five years, the global network of computers, telephones and televisions, as well as other business and consumer electronics devices, has increased its information transmission capacity by about two million times. Computer power doubles approximately every 18 months according to the Moore's Law [16]. Several economies, from different countries, have opened their markets to trade and capital. Is this their way into deeper poverty?

Decisions on the basis of which the developmental changes are to be made should focus on both risk and the essence of functional differentiation. Danger always signifies something external that happens independently of our will, while at risk we examine the possible shortcomings of our decisions. Unwillingness to the technique can compromise its innovation power. With the advanced "functional differentiation", the structure of society becomes more contingent. In social development, targeted, we are increasingly setting ourselves up for contingency, relying more and more on science and technologies based on it. As a result, the dimension of the future, while still a contingent factor, increasingly becoma the decisive determinant of behavior in the present, becoming more certain. The increasing strategic importance of science and technology for the social dynamism means, whether we like it or not, that our behavior is increasingly oriented toward the future. By entering both the "knowledge society" and "risk society", the dimension of the future becomes central through the greatest possible measure of risk inclusion in development decisions.

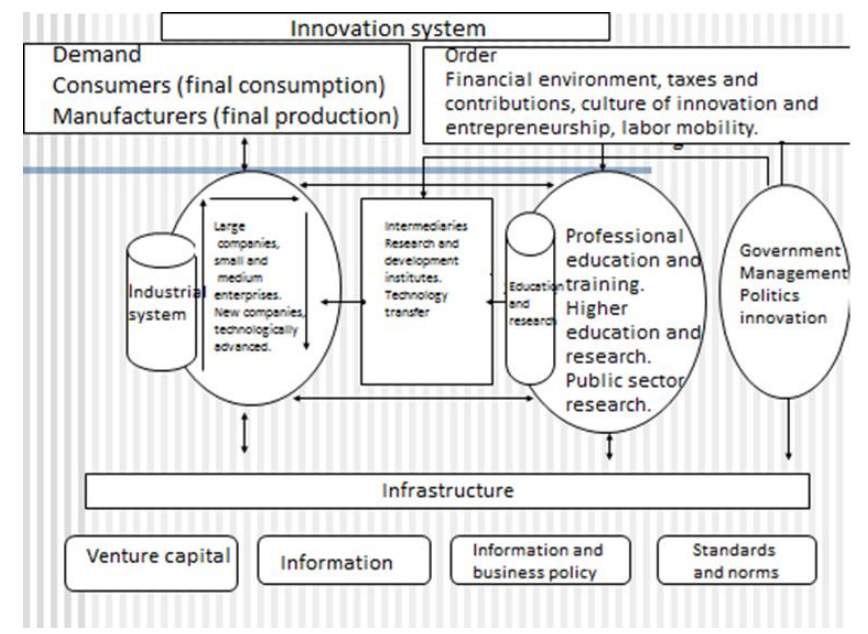

Figure 2 Model of the holistic approach to innovation [3] 
We point out the entrepreneurship from a position of innovation and a tendency to accept the risks and overcome the existing and the achieved. It turns out that they are the entrepreneurs and innovators, people who are not risk averse. In order to do better and differently, the entrepreneurs must own the so-called "distinctive competence." Distinctive competency means a unique insight into the market, technology and management to compete with the competitors [14].

There are important differences between the entrepreneurship and management. Management is based on combining factors of production to produce, while entrepreneurship is based on combining factors of production to initiate a change. Management is a phenomenon of continuity and entrepreneurship is a phenomenon of discontinuity. The essence of entrepreneurship is change, and nothing today is as permanent as change [10].

The new branches of high technology characterize the small businesses in the first stages of development. These are the companies that usually combine the entrepreneurial idea, capital and management skills in an individual.

During the 1990s, a new phenomenon of the so-called "enterprise kindergarten". These are companies that use the Internet as infrastructure, whose founders are younger people with no business experience.

\footnotetext{
* Top list of innovations for the last 35 years without being in the medical field. Internet created in 1969 as an Arpanet- military communications network. Mobile Phone, PC, Optical Fiber - Fiber Phone Calls Have Been Cheap Wherever You Are Calling Other than from Serbia, Email, GPS, Laptop, CD / DVD, Digital Camera, RFID, Microelectromechanical Systems, DNA Imprint, Airbag, ATM , Advanced Batteries, Hybrid Car, OLED, Display Panels, High Definition TV, Nanotechnology, Spacecraft, Flash Memory (micro version of computer disk drive), Voice Messages, Hearing Aids, Low bandwidth radio, drone, smartphone. Smart technology, $3 D$ printer ...
}

The concept of Industry 4.0 in the world has existed since the fall of 2011, when it was defined as a model for the development of Germany as the dominant industrial economy today. It basically, in a new way, defines the concept of industrial automation (internet of things, cloud technologies, cyber systems). Germany has been a leader in this field and the national programs of Industry 4.0 in the US, England, Japan, China and the EU indicate that there is no unique approach to this program in the world, but each country, based on its national priorities, defines its model as a strategic technological development framework. Serbia currently has a defined framework for the National Industry 4.0 Program, set up through the US-EU-JapanSerbia Technology Summit, held in 2016 and 2017, on the global topics: National Industrial Policy for Industry 4.0 (2016) and Intelligent and Smart Products in the context of Industry 4.0 (2017), which is an opportunity for the other countries in the region to become involved in this Program.

In order to accomplish its innovative mission, the company has at its disposal Internet (IoT) as well as the cyber-physical structures. Business structures set the boundaries between the cyber and real physical world. Continuity of action is generally related to the space and time.

Robotics, telecommunications, and computers are the paradigm of information technology. Robotics, but also selfregulating supporting technical and technological systems and processes based on knowledge, solutions based on the "cloud computing" and Internet data of a functioning system, affirm at least two positive aspirations. In the physical sense, the products are created, while in the digital realm, the processes of creative action take place in order to improve the characteristics that have the quality and dominant information in the field to which the action relates. 


\section{EVOLUTION AND DIGITAL PROCESSES}

We are witnessing the transition of an evolutionary change in the knowledge economy into an economy of many sizes, definitions and frequent changes [14]. Evolutionary appearance is a natural continuation of previous relationships in the world of work and an introduction to the digital Darwinism.

The evolutionary further development of the system-oriented management science as a generic sum of knowledge starts from the view that both the enterprise and its environment are subject to similar processes of development and principles of action as we can determine in the natural evolution [12].

There are a number of moves in the science of enterprise economics that point to an evolutionary direction that underlies the concepts and methods that emerged within the organizational development movement. So nothing would be easier to push the evolutionary conception of management aside without comment and without explanation. But the concept of evolutionary management goes far beyond spiritually related attempts in the enterprise economics, as it relies in its implications on a not entirely new theory of social systems that goes far beyond enterprise and shows clear differences with respect to the dominant perceptions in most economic and social scientific discipline, and which, in recent years, has undergone a systematic treatment within the framework of some not yet well known works.

The new non-industrialized economies have emerged, and will characterize the coming times. Every economy will strive to create elements of its protection through the power of appearances or through the laws of defense.

We believe that today's markets are more obsessed with events (European Union, Britain, North Korea, Syria, Iraq ... and the Russia-US and China-US relations) than the geopolitical risks. We are sure that there will be the periodic changes and moods, which will disturb the behavior of the market, which will be compounded by more serious geopolitical risks, weakened capacities of institutions and policies based on fear, regardless the possible benignity of political events and changes. In an evolutionary sense, if the market eliminated feudalism, then the digitalism would certainly eliminate the classic market. In fact, we point out that the evolutionary processes and changes are aided by the digitization at the speed of acceleration level The second derivation of the velocity vector [12] (The second derivation of the velocity vector).

The intellectualization of the production process has happened to us. Technological culture dominates with the work culture as a prerequisite of all other cultures. We need a viable competition, which is manifested in a set of relevant criteria that provide a guidance regarding the competitive nature of the market, in order to formulate and execute the competition policy. The structure-management-results trajectory emerged in the industrial economy and defined a representative set of competitiveness criteria [11].

In the evolutionary terms, the collaborative R\&D focuses on applications related to the industry profiles, manufacturing, services, and virtual systems in the areas of autonomous process control, status monitoring, and predictive technical and technological maintenance and enhancement. Other focal points are IoT (Internet-of-Things) platforms, digital infrastructures and standards for connecting elements of the system structure to the higher-level systems or peripherals.

Digital technologies: IoT, robotics, cloud computing, cyber physical systems and big data are the key in implementing Industry 4.0. Industry 4.0 involves the complete digi- 
tization of all production processes and implementation of the aforementioned digital technologies when creating an idea of a product, product engineering, production organization, production realization, process control and the provision of industrial services.

The new strategies are needed, without imitation and recipes, which should be the result-oriented with respect to both economic and social goals. Corresponding to the philosophy of the basic needs is the strategy of normative economics, which starts from an economic analysis that presents proposals or claims related to "what should be" rather than "what is." [2]. We can see that there are contradictions with the positive economy that deals with the economy as it is. Essentially, a normative economy is derived from a positive economy and some estimates of what the goals of society should be. It is possible to challenge the claims of a normative economy if there is disagreement with subjective assessments and presented analyzes [7]. However, the Evolutionary Approach in Economics becomes generally accepted and with it, at the level of development achieved, the digital Darwinism has occurred to us.

Along with discussions on policies and directions for growth and development, the need to respond as a form of reconciling microeconomic and macroeconomic paradigms is indispensable. The new classical macroeconomics increasingly corresponds to the "new microeconomics". The new classical macroeconomics insists on reformulation in the narrow sense of the orthodox classical economy. The basis of reformulation is the concept of rational expectations combined with the concept of the natural rate of unemployment resulting from the effective equilibrium in the market. The starting point is that any attempt to stabilize output or employment below or above the natural unemployment rate through fiscal or monetary policy will prove ineffective and will not change the value of real variables in the long or short term. The basis of this paragraph is the view that there is no compromise between the inflation and unemployment even in the short term, that is, both the aggregate supply curve in the short term and the Phillips curve in the short term are vertical. Whatever is properly seen as a development of monetarism, which in turn is both a development strategy and growth strategy, these views abandon the arguments of leading monetarists who argue that the demandside economic policies will result in deviations of output and unemployment from the natural short-term unemployment rate. In this regard, it is shown that the new classical macroeconomics seeks to show the futility of Keynesian demand management policy and instead focuses on supply economies [7].

Societies and their economies that are on the verge of hopelessness and chaos, facing the escape of people and capital, must create strategies to strengthen institutionalism and institutions if they do not want to enter the chaos that is absolute. Capital does not flow to those countries from which capital escapes. The question we might ask is: do we and how much do we need the role of social, political and economic organizations in determining economic events?

The free-market phrase must be abandoned, as it has been proven that the markets and media everywhere in the world, and even the speculative economy, are free only as much as they are allowed. Too much openness to an under-resilient, underdeveloped and under-developed economy is more dangerous than too closed. The degree of openness of an economy can be approximated by the relative size of its international trade sector relative to the gross national product. And that is why we need the institutions, not only as a satisfaction to the "commanders" of the capital world, but as a controlling factor to ensure their functioning. Accumulation is created in certain territories, 
and often there is an extreme tendency of local politicians, as a rule, economically illiterate, to use it in those same territories, even if such use is not as rational as it would be in another territory. Therefore, removing territorial barriers to asset the circulation is, in fact, a prerequisite for creating a financial market in general and making the investment decisions.

\section{DIGITAL DETERMINANTS OF THE FUTURE}

Digital Darwinism is an era in which technology and societies are evolving faster than companies can adapt. Today we are looking at the modern development of a company through its ability to transform digitally in the sequence of process actions and the achieved level of working culture, application of technology and behavior of society to the development itself. An enterprise with advanced digital technologies is becoming a living organism capable of evolving and thus acquiring the characteristic of a self-learning, adaptive and self-organizing system. In order for digital transformation to be successful, it is necessary to realize the process of adapting and actively re-acting the enterprise to its changing environment. The needs of the users are decisive for the behavior of the company in terms of profiling and production and capacity. These needs are diverse and unequal, requiring a differentiated approach to choosing a business orientation. Businesses are today adapting to the user through code halos, which gives them the ability to map behavior. The state of mind of the company precisely determines the ability of technical and technological innovative development. Research shows that businesses that have embraced the processes of digital transformation and embraced the existence of digital Darwinism have far outpaced competition and entered the desired future much earlier. Businesses that have succeeded in overcoming the process of digital Darwinism have been able to achieve a significant increase in value, especially in the secondary economy.

In a digitized enterprise, it is necessary to design and process the algorithmic process and communication with precision and set up an intelligent interaction of techno-technological processes with the physical, digital and virtual world. In addition to this "networked" fact, in addition to the installed digital machines, equipment and technological systems, the digital enterprise should also have its enterprise-oriented software related to business processes, the Internet and communication infrastructure, the security system and the accompanying digitized service organizations. Software solutions can be developed in their own environment, but most companies purchase them from specialized vendors. The current development of Industry 4.0, back in the 1980s, was preceded by CIM, CAM and CAD, later by SAP and SAIGE, but due to their technological level, they could not capture the overall digitization of the business.

The Digital Darwinism, we can write freely, is in the milieu of many companies. Many have developed, lasted and survived, while those that failed to adapt to the new conditions have disappeared from the market and economic environment. Starting from the specific intelligence of the enterprise, immanent to man, as the basic management lever, to manage things and processes with the aim of producing output, based on evolutionary principle, in order to produce output.

By formulation the principle of indeterminacy, we transform the natural laws into statistical laws. Today we are becoming aware of a world whose pillars have lost the solidity of granite institutions (laws), dominated by the bundles of probabilities of smaller or larger ones, and to which the notion of certainty, or ideal probability, can be more adequately represented by an infinite decimal fraction of $0.9999 \ldots$ one imaginary barrier that di 


\section{CONCLUSIONS}

vided science into the "real" or exact and "verbal" - social was demolished. Fortunately, the whole objective continuum becomes once again a whole in which homogeneous laws operate, differing in the intensity and structure, and not in their nature.

The intellectual climate favored the emergence of a knowledge-based economy. And did knowledge of economics alone favor the development of a knowledge economy? Let's go back to the mainland. There was a focus on sharp semantic barriers between the professions, on building as efficient an interdisciplinary scientific method of managing complex, stochastic systems, and on developing a general theory of management and communication.

Everyone fears the future, because of its absolute uncertainty, regardless of the projected outcomes!? Economic globalization it has four forms, namely the flow of goods and services, the so-called free trade, human flow and migration, capital flow and technology. The problem is its unidirectionality and no equal partnership and equal cooperation. The dilemma is individualism or collectivism, or intellectual cooperation? The second dilemma is: the artificial intelligence and collective brain!? One will not face the possibility of his or her being excommunicated in the decision-making processes. The inauguration of security systems and their development is a parallel response to the digital developments to keep them under control. Networking is one of the basic features of digitalization. For example, the absence of economic and market integration of agriculture, which is the basis of industrial production, but also of other industries that use the agricultural and agricultural products, is evident to lead to a significantly higher output than the needs of the domestic market, which, we assume, implies an export orientation, but also significant product restructuring.
Research mobility carries with it the message of generating results. The scientific basis is determined by personnel inputs and the absence of objective selection on the one hand and the level of development of the economy on the other. Verification of the results of the R\&D activity requires a developed material base, which will return to the scientific system challenging tasks that the scientific system will have to solve. The state, on the other hand, should have an integrating role in the management of knowledge on a broad economic basis creating the technological and innovation policies as an integral part of the overall economic policy. This includes:

1. Refocusing specific subjects and adapting technological and innovation policy instruments. In doing so, they gain the new importance of policies to promote the research collaboration, accelerate enterprise networking and clustering, encourage institutional connections, diffuse technology and increase employee mobility.

2. Ensuring the framework conditions for innovation management, where particular attention should be paid to: competitiveness policies to increase competitiveness through innovation but also to accelerate collaborative research; education and training policies to develop the necessary human capital; regulatory reform policies to reduce administration and institutional rigidity; and financial and fiscal policies, communications policies, foreign investment, and trade and regional policies.

Technology is involved in all organizational activities, and its rapid changes make technological change a significant factor in almost every innovation of an organization.

We are witnessing the transition of an evolutionary change in the knowledge economy into an economy of many magnitudes, definitions and frequent changes [14]. Evolutionary appearance is a natural continuation of previous relationships in the world of 
work and an introduction to digital Darwinism.

The experiences of previous periods and the needs of the people, scientific technical and technological organizational, managerial as well as total social achievements, enable, to a large extent, a look into the movement on the world economic scene in the near and far future, as well as the appearance and change of a great evolutionary change and its duration. New non-industrialized economies have emerged and will emerge that will characterize the coming time. Every economy will strive to create elements of its protection through the power of appearances or through the laws of defense.

We can freely write that today's markets are more obsessed with events (the European Union, Britain, North Korea, Syria, Iraq $\ldots$ and the Russia-US and China-US relations) than geopolitical risks. We are sure that there will be periodic changes and moods, which will disturb the behavior of the market, which will be compounded by more serious geopolitical risks, weakened capacities of institutions and policies based on fear, regardless of the possible benignity of political events and changes.

Modern production and business systems to be able to function effectively should create a platform for information exchange at every moment of its appearance and operation. Then intelligent structures will be able to communicate with other systems, all for the purpose of insight and access through decentralized control. Modern industrial processes and smart organizations, without the incorporation of intelligent technologies, would not work. There would be a radical discontinuity in the performance of their business missions, crises and system downfall.

The economies of developing countries are caged by global trends that we have defined as an expression of power and power.
With the increase of the cage, does the degree of freedom increase or are you still in the cage!? There is always the question of human profitability in cyberspace.

\section{REFERENCES}

[1] Balaz Z. Meštrović. K. (2018) Polytechnic Cognitive Cybernetics, Technical University of Zagreb, p. 10.

[2] Davidson M., (1995) The Transformation of Management, Macmilan Business, London, p. 187., p. 196.

[3] Lana Hopkinson EU Innovation Expert; Modified based on presentation in the Serbian Chamber of Commerce

[4] Lester Thurow, an economist at MIT, argues in his book The Future of Capitalism that Tectonic Shifts Break the Old Rules of Capitalism.

[5] Milačić, V.: Production Systems and Technology of Innovation, Belgrade 1976, p. 131.

[6] Fundamentals of Science Policy in the SFRY, Yugoslav Review 7-8/74, p. 269 in Ristić Ž. Knowledge Management, Etiquette Graphic Center, Belgrade, 2001.

[7] Pearce W. D., (2003) Modern Economics 4 ed, MacMillan, p. 307-308

[8] Stoner J., Freeman E., Management, $4^{\text {th }}$ ed., 1989, Prentice Hall, New York

[9] Todosijevic R. Todosijevic Lazovic S., (1-2.09. 2017) Institutionalism and Digital Darwinism, Andrić Grad, Scientific Conference, "Economics Today" Collected Papers.

[10] Todosijević Lazović S,. New Production Programs, Growth of Production Capacity and Economic Efficiency of Enterprises, (2010) Doctoral Thesis, University of Novi Sad, p. 3. 
[11] Todosijević R. (2010) Strategic Management, Volume 1. Faculty of Economics Subotica, p.255-268, p.530.

[12] Todosijević R. (2010) Strategic Management, Volume 2. Faculty of Economics Subotica, p. 11

[13] Todosijevic R. Todosijevic Lazovic S., (2019), Digitization and Evolutionary Economics in the Cyber Milieu, EkonBiz, International Scientific Conference, Faculty of Business Economics Bijeljina.

[14] Zelenovic D., (2011) Intelligent Business, Basic Technology of a Serious Society, Prometheus, p. 95.
[15] Todosijevic Lazovic S., Milacic S Todosijevic R. V. (2016) Scientific research and knowledge horizons, Acta Economica no. 24, Banja Luka. p.112.

[16] Gordon Moore, co-founder of American Intel

[17] The avalanche of future phobic books hints, no less, than the end of the old economic order. Let's look at the titles of books: "End of Abundance", "End of Labor", "Death of Inflation", "Death of Compe-tition", End of Geography ","Death of Money "or even" Death of Economic Science". 